HEP/123-qed

\title{
Manipulation and readout of spin states of a single-molecule magnet by a spin-polarized current
}

\author{
Hai-Bin Xue, ${ }^{1, *}$ Jiu-Qing Liang, ${ }^{2}$ and Wu-Ming $\mathrm{Liu}^{3}$ \\ ${ }^{1}$ Key Laboratory of Interface Science and Engineering in Advanced Materials, \\ Ministry of Education, Taiyuan University of Technology, Taiyuan, Shanxi 030024, China \\ ${ }^{2}$ Institute of Theoretical Physics, Shanxi University, Taiyuan, Shanxi 030006, China \\ ${ }^{3}$ Beijing National Laboratory for Condensed Matter Physics, \\ Institute of Physics, Chinese Academy of Sciences, Beijing, 100190, China.
}

(Dated: September 7, 2021)

\begin{abstract}
Single-molecule memory device based on a single-molecule magnet (SMM) is one of the ultimate goals of semiconductor nanofabrication technologies. Here, we study how to manipulate and readout the SMM's two spin-state of stored information that characterized by the maximum and minimum average value of the $Z$-component of the total spin of the SMM and the conductionelectron, which are recognized as the information bits "1" and " 0 ". We demonstrate that the switching time depends on both the sequential tunneling gap $\varepsilon_{s e}$ and the spin-selection-rule allowed transition-energy $\varepsilon_{\text {trans }}$, which can be tuned by the gate voltage. In particular, when the external bias voltage is turned off, in the cases of the unoccupied and doubly-occupied ground eigenstates, the time derivative of the transport current can be used to read out the SMM's two spin-state of stored information. Moreover, the tunneling strength of and the asymmetry of the SMM-electrode coupling have a strong influence on the switching time, but that have a slight influence on the readout time that being on the order of nanoseconds. Our results suggest a SMM-based memory device, and provide fundamental insight into the electrical controllable manipulation and readout of the SMM's two spin-state of stored information.
\end{abstract}

PACS numbers: 75.50.Xx, 85.65.+h, 82.37.Gk

*Electronic address: xuehaibin@tyut.edu.cn 


\section{INTRODUCTION}

The realization of memory device based on single-molecule magnet (SMM) is currently one of the ultimate goals of semiconductor nanofabrication technologies. In particular, the SMM memory devices provide the final density-limit of magnetic storage media [1 5 ]. Consequently, the single-molecule magnet (SMM), as a smallest magnetic bistable system [3, 4, 6 -12], has been suggested as a promising candidate for the information storage and processing due to its chemical tunability and scalability [13]. A fundamental step towards realization of the SMM-based memory devices is the manipulation and detection of spin states of the SMM. In the nanoscale devices, a local magnetic field is required to manipulate the spin degree of freedom. In resent years, using electric fields instead of magnetic fields to manipulate the spin states of the nanomagnets (i.e., the SMM, magnetic atoms on surfaces and magnetic molecules) have become the subject of intensive experimental [14 $[22]$ and theoretical [23-40] investigations due to the electric fields being easily focused and shielded within a small volume.

The electric-field manipulation of spin states of a SMM can be realized by transferring the spin angular momentum of conduction electrons to the SMM via the the spin transfer torque. For example, the switching of spin states in the SMM can be controlled with an external spin-polarized current through the SMM, which is coupled with two electrodes either one 23, 28] or both of them being ferromagnetic [24 26, 29, 39], or applied by a spin bias voltage [27]. The giant-spin orientation of the SMM can be determined by the direction of spinpolarized current [23, 28, 29]. From the SMM-based memory device point of view, there are at least two aspects of the bias-voltage-driven switching of the SMM's spin-state that should be considered. Firstly, what are the essential factors that governed the switching time of the SMM's two spin-state of stored information? Secondly, how to read out the SMM's two spin-state of stored information? The previous works are mainly focused on the first aspect, for example, the influences of the bias voltage, the spin polarizations of the source and drain electrodes, and the SMM's internal level structure on the switching processes of the SMM's spin states. However, the dependence of the switching time and the readout time on the SMM's ground eigenstate have not yet been revealed. In particular, the readout scheme of and the readout time of the SMM's two spin-state of stored information are still some open issues and require further studies. 
In this work, we study the influences of the SMM's ground eigenstates on the switching time and the readout time, and discuss how to manipulate and read out the SMM's two spin-state of stored information. It is numerically demonstrated that the minimum switching time, which is strongly affected by the tunneling strength of and the asymmetry of SMMelectrode coupling, can be observed when the energy eigenvalues of the SMM's two singlyoccupied eigenstates approach that of the unoccupied or doubly-occupied ones. In particular, in the cases of the unoccupied and doubly-occupied ground eigenstates, the effective readout scheme of the SMM's two spin-state of stored information is proposed by turning off the external bias voltage. The corresponding readout time of the SMM's two spin-state of stored information is of the order of nanoseconds. These results suggest the feasibility of an individual SMM as single-molecule memory device.

\section{MODEL AND FORMALISM}

\section{A. Hamiltonian of a single-molecule magnet coupled to two electrodes}

The single-molecule memory device consists of a SMM weakly coupled to the two ferromagnetic leads, as shown schematically in Fig. 1. Here, we consider a prototypical SMM $\mathrm{Mn}_{12}\left(\mathrm{Mn}_{12}\right.$-acetate) [6, 7, 9, 11], which has total spin $S=10$ and an anisotropy barrier of $6 \mathrm{meV}$. The SMM Hamiltonian can be described by

$$
H_{\mathrm{SMM}}=\left(\varepsilon_{\mathrm{LUMO}}-e V_{g}\right) \hat{n}+\frac{U}{2} \hat{n}(\hat{n}-1)-J \vec{s} \cdot \vec{S}-K_{2}\left(S_{z}\right)^{2},
$$

Here, the first and second terms describe the lowest unoccupied non-degenerate molecular orbital (LUMO) with on-site energy $\varepsilon_{\text {LUMO }}$, which can be tuned by a spin-independent gate voltage $V_{g} . \quad \hat{n} \equiv d_{\uparrow}^{\dagger} d_{\uparrow}+d_{\downarrow}^{\dagger} d_{\downarrow}$ with $d_{\sigma}^{\dagger}\left(d_{\sigma}\right)$ being the creation (annihilation) operator of an electron with spin $\sigma$ for the LUMO. $U$ is the on-site Coulomb repulsion between two electrons in the LUMO. The third term denotes the exchange coupling between the conduction-electron spin $\vec{s} \equiv \sum_{\sigma \sigma^{\prime}} d_{\sigma}^{\dagger}\left(\vec{\sigma}_{\sigma \sigma^{\prime}}\right) d_{\sigma^{\prime}}$ and the giant spin $\vec{S}$ of the SMM, where $\vec{\sigma} \equiv$ $\left(\sigma_{x}, \sigma_{y}, \sigma_{z}\right)$ is the vector of Pauli matrices. The forth term is the anisotropy energy of the SMM, whose easy-axis is $Z$-axis $\left(K_{2}>0\right)$, thus, the spin is quantized along the $Z$-axis.

The spin relaxation in the two electrodes is assumed to be sufficiently fast, thus, their electron distributions can be modeled as non-interacting Fermi gases and the Hamiltonian 
reads

$$
H_{\text {electrodes }}=\sum_{\alpha \mathbf{k} s} \varepsilon_{\alpha \mathbf{k} s} a_{\alpha \mathbf{k} s}^{\dagger} a_{\alpha \mathbf{k} s}
$$

where $a_{\alpha \mathbf{k} \sigma}^{\dagger}\left(a_{\alpha \mathbf{k} \sigma}\right)$ is the creation (annihilation) operator of an electron with energy $\varepsilon_{\alpha \mathbf{k} \sigma}$, momentum $\mathbf{k}$ and spin $s$ in $\alpha(\alpha=L, R)$ electrode, with the index $s=+(-)$ denoting the majority (minority) spin states with the density of states $g_{\alpha}^{s}$. The electrode polarization is characterized by the orientation of the polarization vector $\mathbf{P}_{\alpha}$ and its magnitude is defined as $P_{\alpha}=\left(g_{\alpha}^{+}-g_{\alpha}^{-}\right) /\left(g_{\alpha}^{+}+g_{\alpha}^{-}\right)$. Here, the polarization vectors $\mathbf{P}_{L}$ (left electrode) and $\mathbf{P}_{R}$ (right electrode) are parallel or antiparallel to the spin quantization $Z$-axis. In the parallel case, spin-up $\uparrow$ and spin-down $\downarrow$ are defined to be the majority spin and minority spin of the ferromagnetic electrode, respectively. The tunneling Hamiltonian between the SMM and the two electrodes is thus described by

$$
H_{\text {tunneling }}=\sum_{\alpha \mathbf{k} \sigma}\left(t_{\alpha \mathbf{k} \sigma} a_{\alpha \mathbf{k} \sigma}^{\dagger} d_{\sigma}+\text { H.c. }\right),
$$

In the ferromagnetic electrode case, the spin-dependent tunneling rate is given as $\Gamma_{\alpha}^{\sigma}=$ $2 \pi\left|t_{\alpha}\right|^{2} g_{\alpha}^{\sigma}=\left(1+\sigma P_{\alpha}\right) \Gamma_{\alpha} / 2$ and $\Gamma_{\alpha}=\Gamma_{\alpha}^{\uparrow}+\Gamma_{\alpha}^{\downarrow}$, where the tunneling amplitudes $t_{\alpha}$ and the density of the state $g_{\alpha}^{\sigma}$ are assumed to be independent of wave vector and energy. While in the normal-metal electrode case, $P_{\alpha}=0$ and then $\Gamma_{\alpha}^{\uparrow}=\Gamma_{\alpha}^{\downarrow}=\Gamma_{\alpha} / 2$. In the case of the polarization vector $\mathbf{P}_{\alpha}$ being antiparallel to the spin quantization $Z$ axis, the electrode- $\alpha$ spin polarization rate $P_{\alpha}$ takes a negative value.

\section{B. Energy eigenstates of the isolated SMM}

In terms of the four electron states of the LUMO $|0,0\rangle_{\mathrm{LUMO}},|\uparrow, 0\rangle_{\mathrm{LUMO}},|0, \downarrow\rangle_{\mathrm{LUMO}}$, $|\uparrow, \downarrow\rangle_{\mathrm{LUMO}}$, and the spin states of the SMM $|m\rangle_{\mathrm{GS}}(m=-S,-S+1, \cdots, S-1, S)$, the unoccupied and doubly-occupied eigenstates can be expressed as

$$
|0, m\rangle=|0,0\rangle_{\mathrm{LUMO}}|m\rangle_{\mathrm{GS}}, m=-S,-S+1, \cdots, S-1, S
$$

and

$$
|2, m\rangle=|\uparrow, \downarrow\rangle_{\mathrm{LUMO}}|m\rangle_{\mathrm{GS}}, m=-S,-S+1, \cdots, S-1, S,
$$

respectively. In the singly-occupied case, the eigenstates of isolated SMM have the following two branches [23]

$$
|1, m\rangle^{ \pm}=a_{m}^{ \pm}|\uparrow, 0\rangle_{\mathrm{LUMO}}\left|m-\frac{1}{2}\right\rangle_{\mathrm{GS}}+b_{m}^{ \pm}|0, \downarrow\rangle_{\mathrm{LUMO}}\left|m+\frac{1}{2}\right\rangle_{\mathrm{GS}},
$$


with

$$
\begin{gathered}
a_{m}^{ \pm}=\frac{J \sqrt{S(S+1)-m^{2}+1 / 4}}{2 \sqrt{\Delta E(m)} \sqrt{2 \Delta E(m) \mp\left(2 K_{2}-J\right) m}}, \\
b_{m}^{ \pm}=\mp \frac{\sqrt{2 \Delta E(m) \mp\left(2 K_{2}-J\right) m}}{2 \sqrt{\Delta E(m)}} .
\end{gathered}
$$

where $\Delta E(m)=\left[K_{2}\left(K_{2}-J\right) m^{2}+(J / 4)^{2}(2 S+1)^{2}\right]^{1 / 2}$ and $m=-S+1 / 2,-S+3 / 2$, $\cdots, S-3 / 2, S-1 / 2$ that being the magnetic quantum-number of the total spin of the SMM and the conduction-electron. While in the $m= \pm(S+1 / 2)$ cases, the two singlyoccupied branches are $|1, S+1 / 2\rangle=|\uparrow, 0\rangle_{\mathrm{LUMO}}|S\rangle_{\mathrm{GS}}$ for $m=S+1 / 2$, and $|1,-S-1 / 2\rangle=$ $|0, \downarrow\rangle_{\mathrm{LUMO}}|-S\rangle_{\mathrm{GS}}$ for $-S-1 / 2$. The corresponding energy eigenvalues are given by

$$
\begin{gathered}
\varepsilon(0, m)=-K_{2} m^{2}, \\
\varepsilon(2, m)=2\left(\varepsilon_{\mathrm{LUMO}}-e V_{g}\right)+U-K_{2} m^{2}, \\
\varepsilon^{ \pm}(1, m)=\varepsilon_{\text {LUMO }}-e V_{g}+\frac{J}{4}-K_{2}\left(m^{2}+\frac{1}{4}\right) \pm \Delta E(m) .
\end{gathered}
$$

Here, for the states $|1, \pm(S+1 / 2)\rangle$, the upper (lower) sign applies if $K_{2}-J / 2$ is positive (negative). It is found from Eqs. (8) and (9) that the energy eigenvalues of the eigenstates $|1, m\rangle^{ \pm}$and $|2, m\rangle$ can be tuned by an applied gate voltage $V_{g}$.

\section{Quantum master equation and dynamics of the density matrix}

Due to the SMM-electrode coupling is sufficiently weak, the sequential tunneling dominates the transitions. The electron tunneling processes are well described by quantum master equation of a reduced density matrix in a set of basis vectors of the SMM's eigenstates. Under the Born approximation and Markovian approximation, the second-order quantum master equation for the reduced density matrix is given by [41]

$$
\dot{\rho}(t)=-i \mathcal{L} \rho(t)-\frac{1}{2} \mathcal{R} \rho(t),
$$

with

$$
\mathcal{R} \rho(t)=\sum_{\sigma=\uparrow, \downarrow}\left[d_{\sigma}^{\dagger} A_{\sigma}^{(-)} \rho(t)+\rho(t) A_{\sigma}^{(+)} d_{\sigma}^{\dagger}-A_{\sigma}^{(-)} \rho(t) d_{\sigma}^{\dagger}-d_{\sigma}^{\dagger} \rho(t) A_{\sigma}^{(+)}\right]+H . c .
$$

where $A_{\sigma}^{( \pm)}=\sum_{\alpha=L, R} A_{\alpha \sigma}^{( \pm)}, A_{\alpha \sigma}^{( \pm)}=\Gamma_{\alpha}^{\sigma} n_{\alpha}^{ \pm}(-\mathcal{L}) d_{\sigma}, n_{\alpha}^{+}=f_{\alpha}, n_{\alpha}^{-}=1-f_{\alpha}$, and $f_{\alpha}$ is the Fermi function of the electrode $\alpha$. The Liouvillian superoperator $\mathcal{L}$ is defined as $\mathcal{L}(\cdots)=$ 
$\left[H_{\mathrm{SMM}},(\cdots)\right]$. Throughout this work, we set $e \equiv \hbar=1$. The differential equation (10) can be solved by the fourth order Runge-Kutta method. Here, the average value of the $Z$-component of the total spin of the SMM and the conduction-electron is defined as $\left\langle S_{z}\right\rangle=\sum_{n, m} m P_{|n, m\rangle}$, where $P_{|n, m\rangle}$ is the occupation probability of the eigenstate $|n, m\rangle$. The maximum value $\left\langle S_{z}\right\rangle_{\max }$ corresponds to the SMM's spin being parallel to the $Z$-axis, whereas the minimum one $\left\langle S_{z}\right\rangle_{\min }$ corresponds to that being antiparallel to the $Z$-axis. Consequently, the SMM's two spin-state of the $\left\langle S_{z}\right\rangle_{\max }$ and $\left\langle S_{z}\right\rangle_{\min }$ are evaluated, respectively, as the two states of the binary code, namely, the spin-up (1) and spin-down (0).

\section{RESULTS AND DISCUSSIONS}

We now study the manipulation and readout of the SMM's two spin-state of the $\left\langle S_{z}\right\rangle_{\max }$ and the $\left\langle S_{z}\right\rangle_{\min }$, and discuss the dependence of the switching time and the readout time on the SMM's ground eigenstate and the tunneling strength of and the asymmetry of SMMelectrode coupling. The typical parameters of a $\mathrm{SMM} \mathrm{Mn}_{12}$ are taken as: $S=10, U=25$, $J=0.2, K_{2}=0.06$ and $k_{B} T=0.4$, where the energy unit is meV. In addition, the bias voltage is assumed to be symmetrically entirely dropped at the SMM-electrode tunnel junctions, i.e., $\mu_{L}=-\mu_{R}=V_{b} / 2$. Here, the initial spin-state is chosen as a mixed states consisting of the SMM's two ground eigenstates with the same occupation probabilities, i.e., $P_{|n,-S\rangle}=P_{|n, S\rangle}=1 / 2(n=0,2)$ or $P_{|1,-S-1 / 2\rangle}=P_{|1, S+1 / 2\rangle}=1 / 2$. Thus, the average value of the SMM's initial spin-state $\left\langle S_{z}\right\rangle_{\text {init }}=0$.

\section{A. Electron tunneling associated with the SMM's spin-state switching}

The spin angular momentum of the conduction electrons can be transferred to the SMM in the electron tunneling processes due to the exchange coupling between electron-spin and the SMM. The electron tunneling channels, which change the quantum number $m$ of the SMM by \pm 1 , can be expressed as follows:

$$
\left\{\begin{array}{c}
|0, m\rangle \stackrel{\text { into: } \uparrow}{\longrightarrow}|\uparrow, 0\rangle_{\mathrm{LUMO}}|m\rangle_{\mathrm{GS}} \stackrel{\text { relax }}{\longrightarrow} \sum_{\nu= \pm} a_{m+\frac{1}{2}}^{\nu}\left|1, m+\frac{1}{2}\right\rangle^{\nu} \stackrel{\text { out: }}{\longrightarrow} \sum_{\nu= \pm} a_{m+\frac{1}{2}}^{\nu} b_{m+\frac{1}{2}}^{\nu}|0, m+1\rangle, \\
|1, m\rangle \stackrel{\text { into: } \uparrow}{\longrightarrow} b_{m}^{ \pm}\left|2, m+\frac{1}{2}\right\rangle \stackrel{\text { out: }}{\longrightarrow} b_{m}^{ \pm}|\uparrow, 0\rangle_{\mathrm{LUMO}}\left|m+\frac{1}{2}\right\rangle_{\mathrm{GS}} \stackrel{\text { relax }}{\longrightarrow} b_{m}^{ \pm} \sum_{\nu= \pm} a_{m+1}^{\nu}|1, m+1\rangle^{\nu}, \\
|2, m\rangle \stackrel{\text { out: } \downarrow}{\longrightarrow}|\uparrow, 0\rangle_{\mathrm{LUMO}}|m\rangle_{\mathrm{GS}} \stackrel{\text { relax }}{\longrightarrow} \sum_{\nu= \pm} a_{m+\frac{1}{2}}^{\nu}\left|1, m+\frac{1}{2}\right\rangle^{\nu} \stackrel{\text { into: } \uparrow}{\longrightarrow} \sum_{\nu= \pm} a_{m+\frac{1}{2}}^{\nu} b_{m+\frac{1}{2}}^{\nu}|2, m+1\rangle,
\end{array}\right.
$$




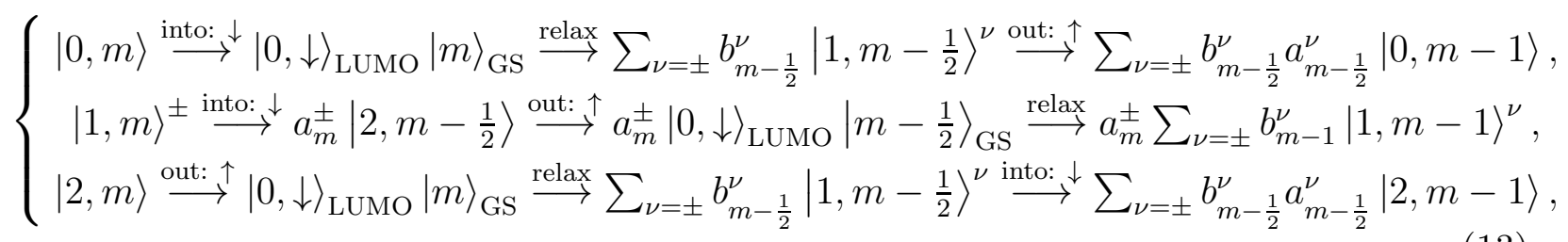

where the notations "into: $\sigma$ " and "out: $\sigma$ " denote the spin $\sigma$ electron tunneling into and out the SMM, respectively. While the notations "relax" describes the electron state of the SMM $|i, j\rangle_{\mathrm{LUMO}}|m\rangle_{\mathrm{GS}}$ relaxing to the corresponding SMM's eigenstates.

The effective switching from the initial spin-state of the $\left\langle S_{z}\right\rangle_{\text {init }}$ to that of the $\left\langle S_{z}\right\rangle_{\max }$ can be realized when the number of spin-up electrons tunneling into the SMM from the source electrode is larger than that of spin-down electrons (i.e., $P_{L}>0$ ), and the number of spin-down electrons tunneling out the SMM and into the drain electrode is not less than that of spin-up electrons (i.e., $P_{R} \leq 0$ ), see Fig. 2(a) and the dashed, dash dotted, and short dotted lines in Figs. 2(b) and 2(c). While the switching from the initial spin-state of the $\left\langle S_{z}\right\rangle_{\text {init }}$ to that of the $\left\langle S_{z}\right\rangle_{\min }$ can take place when $P_{L} \leq 0$ and $P_{R}>0$, i.e., reversing the direction of the bias voltage, see the solid, dotted, and short dashed lines in Figs. 2(b) and 2(c). Consequently, the information can be stored or written onto the SMM's spin-state of the $\left\langle S_{z}\right\rangle_{\max }$ or the $\left\langle S_{z}\right\rangle_{\min }$ by manipulating the direction of the external bias voltage. Moreover, with the increase of the polarization magnitude of the source (drain) electrode $P_{L}\left(P_{R}\right)$, the electron tunneling processes associated with the SMM's spin state switching is accelerated, leading to the decreasing of the corresponding switching time, see Figs 2(b) and 2(c). In the following discussion, we consider a SMM weakly coupled to two ferromagnetic leads with the antiparallel high-spin-polarization configuration, and set $P_{L}=-P_{R}=0.9$.

\section{B. Dependence of the switching time on the SMM's ground eigenstate}

In the SMM system, it is clear from Eqs. (7)-(9) that the ground eigenstates of the SMM can be tuned by the applied gate voltage. For given parameters of the SMM $\mathrm{Mn}_{12}$, the minimum energy eigenvalue of the doubly-occupied eigenstate $\varepsilon_{|2, S\rangle}$ decreases faster than that of the singly-occupied eigenstate $\varepsilon_{|1, S+1 / 2\rangle}$, whereas the minimum energy eigenvalue of the unoccupied eigenstate $\varepsilon_{|0, S\rangle}$ are independent of the applied gate voltage, see Fig. 3. Here, we focus on the region of energy-level crossing between the singly-occupied and doublyoccupied ground eigenstates, and that of energy-level crossing between the singly-occupied 
and unoccupied ground eigenstates, see the region enclosed by the circle with dotted line, and study the influences of the LUMO's energy $\varepsilon_{\text {LUMO }}-e V_{g}$ and the bias voltage on the switching time.

In the first region of the energy-level crossing, with increasing the negative value of the $\varepsilon_{\mathrm{LUMO}}-V_{g}$, which corresponds to the transition of the ground eigenstates from the doublyoccupied to singly-occupied eigenstates, the switching time first decreases and then increases, see Figs 4(c) and 4(d). While in the second region of the energy-level crossing, with increasing the value of the $\varepsilon_{\text {LUMO }}-V_{g}$ from negative values to positive values, which corresponds to the transition of the ground eigenstates from the singly-occupied to unoccupied eigenstates, the switching time first also decreases and then increases, see Figs 4(a) and 4(b).

In fact, for the fixed bias voltage and spin polarizations of the source and drain electrodes, the switching time depends on both the sequential tunneling gap $\varepsilon_{\text {se }}$ [42], which is the energy difference between the ground eigenstate of charge $N$ and the first excited eigenstate of charge $N-1$, and the spin-selection-rule allowed transition-energy $\varepsilon_{\text {trans }}$, which is the difference between the energy eigenstates with the electron-number $n$ and $n \pm 1$ satisfying the spin selection rule. Consequently, with increasing the value of the $\varepsilon_{\text {LUMO }}-V_{g}$ from negative values to positive values, the transition of the sequential tunneling gap $\varepsilon_{s e}$ from $\left|\varepsilon_{|1, \pm(S+1 / 2)\rangle}-\varepsilon_{|2, \pm S\rangle}\right|$ to $\left|\varepsilon_{|1, \pm(S+1 / 2)\rangle}-\varepsilon_{|0, \pm S\rangle}\right|$, and the magnitudes of the sequential tunneling gap $\varepsilon_{s e}$ and the transition energy $\varepsilon_{\text {trans }}$ can reach relatively small values in the two energy-level crossing regions, i.e., the energy eigenvalues of the doubly-occupied branch approach that of the two singly-occupied branches, and the energy eigenvalues of the two singly-occupied branches approach that of the unoccupied branch. Particularly, the switching time can reach a minimum value when the sequential tunneling gap $\varepsilon_{s e}$ is approximately equal to zero, which corresponds to the two energy-level crossing points, see Fig. 3 and the $\varepsilon_{\mathrm{LUMO}}-V_{g}=1.0$ and $\varepsilon_{\mathrm{LUMO}}-V_{g}=-26.0$ cases in Fig. 4.

Next, we discuss the influence of the bias voltage on the spin-state switching processes. For the given LUMO's energy $\varepsilon_{\mathrm{LUMO}}-e V_{g}$ and spin polarizations of the source and drain electrodes, with increasing the applied bias voltage, new electron tunneling channels can be opened, which further increase the electron tunneling processes associated with the spinstate switching. The switching processes are thus enhanced rapidly, and the corresponding switching time then decreases, see Fig. 5. However, When the applied bias voltage is larger than a threshold bias voltage that depending on the SMM's internal level structure, all 
tunneling channels are opened, and then the switching time approaches a saturated value. see the $V_{b}= \pm 10$ and $V_{b}= \pm 12$ cases in Fig. 5. Therefore, the switching time decreases with increasing the applied bias voltage at first and approaches a minimum value finally at the so-called saturated bias voltage.

Furthermore, the interchange between the two spin-state of the $\left\langle S_{z}\right\rangle_{\max }$ (i.e., the information bits "1") and the $\left\langle S_{z}\right\rangle_{\min }$ (i.e., the information bits "0") is an important aspect of the error correction of the spin states of stored information. Figure 6 displays the transition from the spin-state of the $\left\langle S_{z}\right\rangle_{\max }$ to the spin-state of the $\left\langle S_{z}\right\rangle_{\min }$ or in the opposite way from

the spin-state of the $\left\langle S_{z}\right\rangle_{\min }$ to the spin-state of the $\left\langle S_{z}\right\rangle_{\max }$. Here, the initial spin-state of the $\left\langle S_{z}\right\rangle_{\max }$ and the $\left\langle S_{z}\right\rangle_{\min }$ are chosen as the steady spin-state in Fig. 4. This operation can be simply carried out by reversing the direction of the writing bias voltage. Therefore, in the $P_{L}=-P_{R}$ case, the direction of the bias voltage can realize the fast switching from a given SMM's spin-state to the spin-state of the $\left\langle S_{z}\right\rangle_{\max }$ or the $\left\langle S_{z}\right\rangle_{\min }$, which is independent of the initial spin-state. Here, it is noted to that in the $P_{L} \neq-P_{R}$ case the values of the spin-state of the $\left\langle S_{z}\right\rangle_{\max }$ and the $\left\langle S_{z}\right\rangle_{\min }$ have a very small difference for the different applied bias voltages. The difference of these values is about the order of $10^{-2}$ for the SMM's parameters considered here.

\section{Readout of spin states of stored information}

Another important processing is how to read out the two spin-state of the $\left\langle S_{z}\right\rangle_{\max }$ and the $\left\langle S_{z}\right\rangle_{\min }$. In order to reduce the influence of the readout processes on the two spin-state of stored information, it requires a small bias voltage and a short readout time. Here, the magnitude of the readout bias voltage is chosen as $V_{b}=0$, namely, the writing bias voltage of the spin-state of the $\left\langle S_{z}\right\rangle_{\max }$ or the $\left\langle S_{z}\right\rangle_{\min }$ is turned off. In this situation, the tunneling processes of conduction electrons are strongly suppressed, and then the occupation probabilities of the spin-state of the $\left\langle S_{z}\right\rangle_{\min }$ and the $\left\langle S_{z}\right\rangle_{\max }$ will undergo a nonequilibrium physical process before they relax to a quasi-equilibrium state. Particularly, the time of the nonequilibrium physical processes is of the order of nanoseconds, see Figs. 7 and 8. We consider the $V_{b}(\mathrm{~W})= \pm 6.0$ case here.

In the initial spin-state of the $\left\langle S_{z}\right\rangle_{\max }$ (or the $\left\langle S_{z}\right\rangle_{\min }$ ), $|1, S+1 / 2\rangle($ or $|1,-S-1 / 2\rangle$ ) has the maximum occupation probability, while $|2, S\rangle$ (or $|2,-S\rangle)$ and $|0, S\rangle$ (or $|0,-S\rangle$ ) 
have a very small occupation probabilities. When the spin-state of the $\left\langle S_{z}\right\rangle_{\max }$ (or the $\left\langle S_{z}\right\rangle_{\min }$ ) relaxes to a quasi-equilibrium state, in the cases of the unoccupied, singly-occupied and doubly-occupied ground eigenstates, the eigenstates $|0, S\rangle$ (or $|0,-S\rangle),|1, S+1 / 2\rangle$ (or $|1,-S-1 / 2\rangle$ ) and $|2, S\rangle$ (or $|2,-S\rangle$ ) have the maximum occupation probabilities, respectively. Therefore, the relaxation of the occupation probabilities of the spin-state of the $\left\langle S_{z}\right\rangle_{\max }$ and the $\left\langle S_{z}\right\rangle_{\min }$ can induce a current, e.g., $|1, S+1 / 2\rangle \stackrel{\text { out: } \uparrow}{\longrightarrow}|0, S\rangle,|1,-S-1 / 2\rangle \stackrel{\text { out: }}{\longrightarrow}$ $|0,-S\rangle,|1, S+1 / 2\rangle \stackrel{\text { into: }}{\longrightarrow}|2, S\rangle$ and $|1,-S-1 / 2\rangle \stackrel{\text { into: } \uparrow}{\longrightarrow}|2,-S\rangle$, although that decreases to zero very rapidly at a few nanoseconds, see Figs. 7 and 8.

In the case of the unoccupied ground eigenstates, in the nonequilibrium relaxation processes, the spin-down electron tunnels out the SMM and into the right-electrode easily due to $\Gamma_{R}^{\downarrow} \gg \Gamma_{R}^{\uparrow}$, which corresponds to the transition $|1,-S-1 / 2\rangle \stackrel{\text { out: }}{\longrightarrow}|0,-S\rangle$. Then, the time derivative of the transport current for the spin-state of the $\left\langle S_{z}\right\rangle_{\min }$ shows much more remarkable variation over time than that for the spin-state of the $\left\langle S_{z}\right\rangle_{\max }$, see Figs. $7($ a) and 7(b). Whereas in the case of the doubly-occupied ground eigenstates, the spin-down electron tunnels into the SMM from the right-electrode easily due to $\Gamma_{R}^{\downarrow} \gg \Gamma_{R}^{\uparrow}$, which corresponds to the transition $|1, S+1 / 2\rangle \stackrel{\text { into: }}{\longrightarrow}|2, S\rangle$, then, the time derivative of the transport current for the spin-state of the $\left\langle S_{z}\right\rangle_{\max }$ shows much more obvious variation over time than that for the spin state of the $\left\langle S_{z}\right\rangle_{\min }$, see Figs. 8(c) and 8(d).

As for the case of the singly-occupied ground eigenstates and the electron tunneling processes being dominated by the transitions between the singly-occupied and unoccupied eigenstates, when the spin-state of the $\left\langle S_{z}\right\rangle_{\max }$ (or the $\left\langle S_{z}\right\rangle_{\min }$ ) relaxes to a quasi-equilibrium state, in the case of the value of $\varepsilon_{|0, S\rangle}$ being slightly larger than that of $\varepsilon_{|1, S+1 / 2\rangle}$, e.g., $\varepsilon_{\mathrm{LUMO}}-V_{g}=0.0$, the occupation probability of the $|1, S+1 / 2\rangle($ or $|1,-S-1 / 2\rangle)$ decreases while that of the $|0, S\rangle$ (or $|0,-S\rangle)$ increases. In this situation, since $\Gamma_{R}^{\downarrow} \gg \Gamma_{R}^{\uparrow}$ the transition $|1,-S-1 / 2\rangle \stackrel{\text { out: }}{\longrightarrow}|0,-S\rangle$ occurs more quickly than $|1, S+1 / 2\rangle \stackrel{\text { out: } \uparrow}{\longrightarrow}|0, S\rangle$, then, the time derivative of the transport current for the spin-state of the $\left\langle S_{z}\right\rangle_{\min }$ shows relatively obvious variation over time than that for the spin-state of the $\left\langle S_{z}\right\rangle_{\max }$, see the $\varepsilon_{\mathrm{LUMO}}-V_{g}=0.0$ case in Figs. $7(\mathrm{c})$ and $7(\mathrm{~d})$. While in the case of the value of $\varepsilon_{|0, S\rangle}$ being much larger than that of $\varepsilon_{|1, S+1 / 2\rangle}$, e.g., $\varepsilon_{\mathrm{LUMO}}-V_{g}=-2.0$, the occupation probability of the $|1, S+1 / 2\rangle$ (or $|1,-S-1 / 2\rangle$ ) further increases while that of the $|0, S\rangle$ (or $|0,-S\rangle$ ) decreases. In this situation, although the transition $|0,-S\rangle \stackrel{\text { into: }}{\longrightarrow}|1,-S-1 / 2\rangle$ occurs more quickly than $|0, S\rangle \stackrel{\text { into: } \uparrow}{\longrightarrow}|1, S+1 / 2\rangle$ due to $\Gamma_{R}^{\downarrow} \gg \Gamma_{R}^{\uparrow}$, the magnitude of the occupation probabilities 
of the $|0,-S\rangle$ and $|0, S\rangle$ is very small. Therefore, the time derivative of the transport current for the spin-state of the $\left\langle S_{z}\right\rangle_{\min }$ does not show an obvious over time than that for the spin-state of the $\left\langle S_{z}\right\rangle_{\max }$, see the $\varepsilon_{\mathrm{LUMO}}-V_{g}=-1.0$ and $\varepsilon_{\mathrm{LUMO}}-V_{g}=-2.0$ cases in Figs. $7(\mathrm{c})$ and $7(\mathrm{~d})$.

Whereas in the case of the singly-occupied ground eigenstates and the electron tunneling processes being dominated by the transitions between the doubly-occupied and singlyoccupied eigenstates, for the spin-state of the $\left\langle S_{z}\right\rangle_{\max }$ (or the $\left\langle S_{z}\right\rangle_{\min }$ ), the occupation probability of the $|2, S\rangle$ (or $|2,-S\rangle$ ) is much larger than that of the $|0, S\rangle$ (or $|2,-S\rangle$ ). In the nonequilibrium relaxation processes, when the value of $\varepsilon_{|2, S\rangle}$ is much larger than that of $\varepsilon_{|1, S+1 / 2\rangle}$, e.g., $\varepsilon_{\mathrm{LUMO}}-V_{g}=-23.0$ and $\varepsilon_{\mathrm{LUMO}}-V_{g}=-24.0$, the occupation probability of the $|1, S+1 / 2\rangle$ (or $|1,-S-1 / 2\rangle$ ) further increases while that of the $|2, S\rangle($ or $|2,-S\rangle$ ) and $|0, S\rangle$ (or $|0,-S\rangle$ ) decrease. Although the spin-down electron tunnels out SMM and into the the right-electrode easily due to $\Gamma_{R}^{\downarrow} \gg \Gamma_{R}^{\uparrow}$, which corresponds to the transition $|2, S\rangle \stackrel{\text { out: }}{\longrightarrow}|1, S+1 / 2\rangle$, the magnitude of the occupation probability of the $|2, S\rangle$ is very small here. Therefore, the time derivative of the transport current for the spin-state of the $\left\langle S_{z}\right\rangle_{\max }$ shows much less obvious variation over time than that for the spin-state of the $\left\langle S_{z}\right\rangle_{\min }$, see the $\varepsilon_{\mathrm{LUMO}}-V_{g}=-23.0$ and $\varepsilon_{\mathrm{LUMO}}-V_{g}=-24.0$ cases in Figs. 8(a) and 8(b). While the value of $\varepsilon_{|2, S\rangle}$ is slightly larger than that of $\varepsilon_{|1, S+1 / 2\rangle}$, e.g., $\varepsilon_{\mathrm{LUMO}}-V_{g}=-25.0$, the occupation probability of the $|1, S+1 / 2\rangle$ (or $|1,-S-1 / 2\rangle$ ) in the nonequilibrium relaxation processes decreases while that of the $|2, S\rangle$ (or $|2,-S\rangle$ ) increases. In this situation, since $\Gamma_{R}^{\downarrow} \gg \Gamma_{R}^{\uparrow}$ the transition $|1, S+1 / 2\rangle \stackrel{\text { into: } \downarrow}{\longrightarrow}|2, S\rangle$ occurs more quickly than that $|1,-S-1 / 2\rangle \stackrel{\text { into: } \uparrow}{\longrightarrow}|2,-S\rangle$, the time derivative of the transport current for the spin-state of the $\left\langle S_{z}\right\rangle_{\max }$ shows relatively obvious variation over time than that for the spin-state of the $\left\langle S_{z}\right\rangle_{\min }$, see the $\varepsilon_{\mathrm{LUMO}}-V_{g}=-25.0$ case in Figs. $8(\mathrm{a})$ and $8(\mathrm{~b})$.

Consequently, in the cases of the unoccupied and doubly-occupied ground eigenstates, the time derivative of the transport current $d I / d t$ as a function of time suggests a possible quantity for the readout of the two spin-state of the $\left\langle S_{z}\right\rangle_{\max }$ and the $\left\langle S_{z}\right\rangle_{\min }$, see Figs. 7(a) and 7(b), and Figs. 8(c) and 8(d). However, in the case of the singly-occupied ground eigenstates, the time derivative of the transport current for the spin-state of the $\left\langle S_{z}\right\rangle_{\max }$ does not show an obvious variation over time than that for the spin-state of the $\left\langle S_{z}\right\rangle_{\min }$ although the switching process might have a relatively small switching time, see Fig. 4, and Figs. 7(c) and 7(d), and Figs. 8(a) and 8(b). 


\section{Dependence of the switching time and the readout time on the SMM-electrode coupling}

To illustrate that the influences of the asymmetry of the SMM-electrode coupling and the corresponding coupling strengths on the switching time and the readout time, we consider here the $\varepsilon_{\text {LUMO }}-e V_{g}=2.0$ case. For the given writing bias voltage and spin polarizations of the source and drain electrodes, the switching time is determined by the number of conduction electrons per unit time tunneling into the SMM from the source electrode, namely, $\Gamma_{L}$, and the number of conduction electrons per unit time tunneling out the SMM and into the drain electrode, namely, $\Gamma_{R}$. Therefore, the SMM-electrode coupling and the corresponding coupling strengths have a strong influence on the switching time, see Fig. 9. Furthermore, although the magnitudes of the $d I / d t$ of the spin-state of stored information over time in the readout process depend on the the asymmetry of the SMM-electrode coupling and the corresponding coupling strengths, the relaxation time is mainly determined by the SMM's internal level structure. Consequently, the asymmetry of the SMM-electrode coupling and the corresponding coupling strengths have a slight influence upon the readout time, which is still of the order of nanosecond, see Fig. 10, and can also be understood in terms of the above mentioned relaxation of the SMM's eigenstates.

\section{CONCLUSIONS}

We have analyzed the feasibility of the SMM-based memory device. Here, the SMM's spin-state of the $\left\langle S_{z}\right\rangle_{\max }$ and that of the $\left\langle S_{z}\right\rangle_{\min }$ are recognized as the information bits "1" and "0", respectively. We have demonstrated that the switching between the SMM's two spin states of stored information (i.e., "1" and "0") can be carried out by reversing the direction of the applied spin-polarized current. This feature may be useful for the error correction of stored information. The switching time, which depends on both the sequential tunneling gap $\varepsilon_{\text {se }}$ and the spin-selection-rule allowed transition-energy $\varepsilon_{\text {trans }}$, can be tuned by the gate voltage. In particular, when the writing bias voltage is turned off, in the cases of the unoccupied and doubly-occupied ground eigenstates, the time derivative of the transport current can be used to read out the SMM's two spin-state of the $\left\langle S_{z}\right\rangle_{\max }$ and the $\left\langle S_{z}\right\rangle_{\min }$. Moreover, the tunneling strength of and the asymmetry of SMM-electrode coupling have a 
strong influence on the switching time, whereas that have a slight influence on the readout time. These results provide fundamental insight into the electrical controllable manipulation and detection of the SMM's two spin-state of stored information, establishing the basis for the application of the SMM in memory device at the single-molecule level.

\section{ACKNOWLEDGMENTS}

This work was supported by the Shanxi Natural Science Foundation of China under Grant No. 201601D011015, Program for the Outstanding Innovative Teams of Higher Learning Institutions of Shanxi, National Key R\&D Program of China under Grant No. 2016YFA0301500, NSFC under Grants Nos.11204203, 11434015, 61227902, 61835013, KZ201610005011, SPRPCAS under Grants Nos. XDB01020300, XDB21030300.

[1] Guo F S, Day B M, Chen Y C, Tong M L, Mansikkamäki A and Layfield R A 2018 Science 362 1400-1403

[2] Sessoli R 2017 Nature 548 400-401

[3] Goodwin C A P, Ortu F, Reta D, Chilton N F and Mills D P 2017 Nature 548 439-442

[4] Guo F S, Day B M, Chen Y C, Tong M L, Mansikkamäki A and Layfield R A 2017 Angew. Chem. Int. Ed. 56 11445-11449

[5] Delgado F and Fernández-Rossier J 2012 Phys. Rev. Lett. 108196602

[6] Heersche H B, de Groot Z, Folk J A, van der Zant H S J, Romeike C, Wegewijs M R, Zobbi L, Barreca D, Tondello E and Cornia A 2006 Phys. Rev. Lett. 96206801

[7] Jo M H, Grose J E, Baheti K, Deshmukh M M, Sokol J J, Rumberger E M, Hendrickson D N, Long J R, Park H and Ralph D C 2006 Nano Lett. 6 2014-2020

[8] Zyazin A S, van den Berg J W G, Osorio E A, van der Zant H S J, Konstantinidis N P, Leijnse M, Wegewijs M R, May F, Hofstetter W, Danieli C and Cornia A 2010 Nano Lett. 10 $3307-3311$

[9] Kahle S, Deng Z, Malinowski N, Tonnoir C, Forment-Aliaga A, Thontasen N, Rinke G, Le D, Turkowski V, Rahman T S, Rauschenbach S, Ternes M and Kern K 2012 Nano Lett. 12 $518-521$ 
[10] E. Burzurí, Zyazin A S, Cornia A and van der Zant H S J 2012 Phys. Rev. Lett. 109147203

[11] Sun K, Park K, Xie J, Luo J, Yuan H, Xiong Z, Wang J and Xue Q 2013 ACS Nano 7 $6825-6830$

[12] Misiorny M, Hell M and Wegewijs M R 2013 Nature Phys. 9 801-805

[13] Bogani L and Wernsdorfer W 2008 Nat. Mat. 7 179-186

[14] Godfrin C, Ferhat A, Ballou R, Klyatskaya S, Ruben M, Wernsdorfer W and Balestro F 2017 Phys. Rev. Lett. 119187702

[15] Godfrin C, Thiele S, Ferhat A, Klyatskaya S, Ruben M, Wernsdorfer W and Balestro F 2017 ACS Nano 113984

[16] Thiele S, Balestro F, Ballou R, Klyatskaya S, Ruben M and Wernsdorfer W 2014 Science 344 $1135-1138$

[17] Vincent R, Klyatskaya S, Ruben M, Wernsdorfer W and Balestro F 2012 Nature 488 357-360

[18] Komeda T, Isshiki H, Liu J, Zhang Y F, Lorente N, Katoh K, Breedlove B K, and Yamashita M 2011 Nat. Commun. 2217

[19] Natterer F D, Yang K, Paul W, Willke P, Choi T, Greber T, Heinrich A J and Lutz C P 2017 Nature $543226-228$

[20] Yan S C, Choi D J, Burgess J A J, Pissarczyk S R and Loth S 2015 Nature Nanotech. 10 $40-45$

[21] Warner B, Hallak F E, Prüser H, Sharp J, Persson M, Fisher A J and Hirjibehedin C F 2015 Nature Nanotech. 10 259-263

[22] Loth S, von Bergmann K, Ternes M, Otte A F, Lutz C P and Heinrich A J 2010 Nat. Phys. $6340-344$

[23] Timm C and Elste F 2006 Phys. Rev. B 73235304

[24] Misiorny M and Barnaś J 2007 Phys. Rev. B 75134425

[25] Misiorny M and Barnaś J 2008 Phys. Rev. B 77172414

[26] Misiorny M and Barnaś J 2009 Phys. Status Solidi B 246 695-715

[27] Lu H Z, Zhou B and Shen S Q 2009 Phys. Rev. B 79174419

[28] Zhang Z Z and Jiang L 2014 Nanotechnology 25365201

[29] Misiorny M and Barnaś J 2013 Phys. Rev. Lett. 111046603

[30] Karlewski C, Marthaler M, Märkl T, Balashov T,Wulfhekel W and Schön G 2015 Phys. Rev. B 91245430 
[31] Delgado F, Palacios J J and Rossier J F 2010 Phys. Rev. Lett. 104026601

[32] Liljeroth P, Repp J and Meyer G 2007 Science 317 1203-1206

[33] Hirjibehedin C F, Lin C Y, Otte A F, Ternes M, Lutz C P, Jones B A and Heinrich A J 2007 Science 317 1199-1203

[34] Gartlanda P and Davidović D 2015 Appl. Phys. Lett. 107172401

[35] Bode N, Arrachea L, Lozano G S, Nunner T S and von Oppen F 2012 Phys. Rev. B 85115440

[36] Delgado F and Rossier J F 2010 Phys. Rev. B 82134414

[37] Comas A B, Chudnovsky E M and Tejada J 2019 J. Phys.: Condens. Matter 31195801

[38] Hymas K and Soncini A 2019 Phys. Rev. B 99245404

[39] Wrześniewski K and Weymann I 2020 Phys. Rev. B 101, 245434

[40] Hymas K and Soncini A 2020 Phys. Rev. B 102045313

[41] Li X Q, Luo J, Yang Y G, Cui P and Yan Y J 2005 Phys. Rev. B 71205304

[42] Aghassi J, Thielmann A, Hettler M H and Schön G 2006 Phys. Rev. B 73195323 


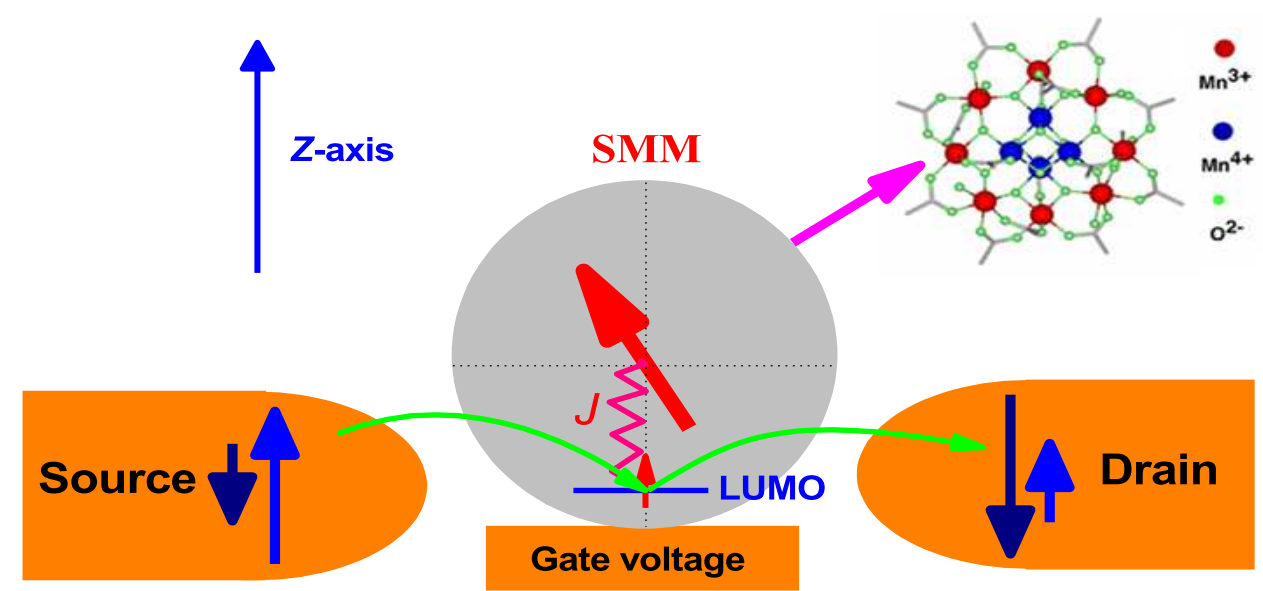

FIG. 1: (Color online) Schematic of a single-molecule memory device consisting of a $\mathrm{SMM} \mathrm{Mn}_{12}$ weakly coupled to the two ferromagnetic electrodes (leads). The SMM's two spin states of the $\left\langle S_{z}\right\rangle_{\max }$ and the $\left\langle S_{z}\right\rangle_{\min }$ are defined as the two states of the binary code, the spin-up (1) and spin-down (0), respectively. Here, $\left\langle S_{z}\right\rangle$, namely, the average value of the $Z$-component of the total spin of the SMM and the conduction-electron is calculated as $\left\langle S_{z}\right\rangle=\sum_{n, m} m P_{|n, m\rangle}$, where $P_{|n, m\rangle}$ is the occupation probability of the SMM's eigenstate $|n, m\rangle$ with $n(n=0,1,2)$ being the electron number and $m$ the magnetic quantum-number of the total spin. 


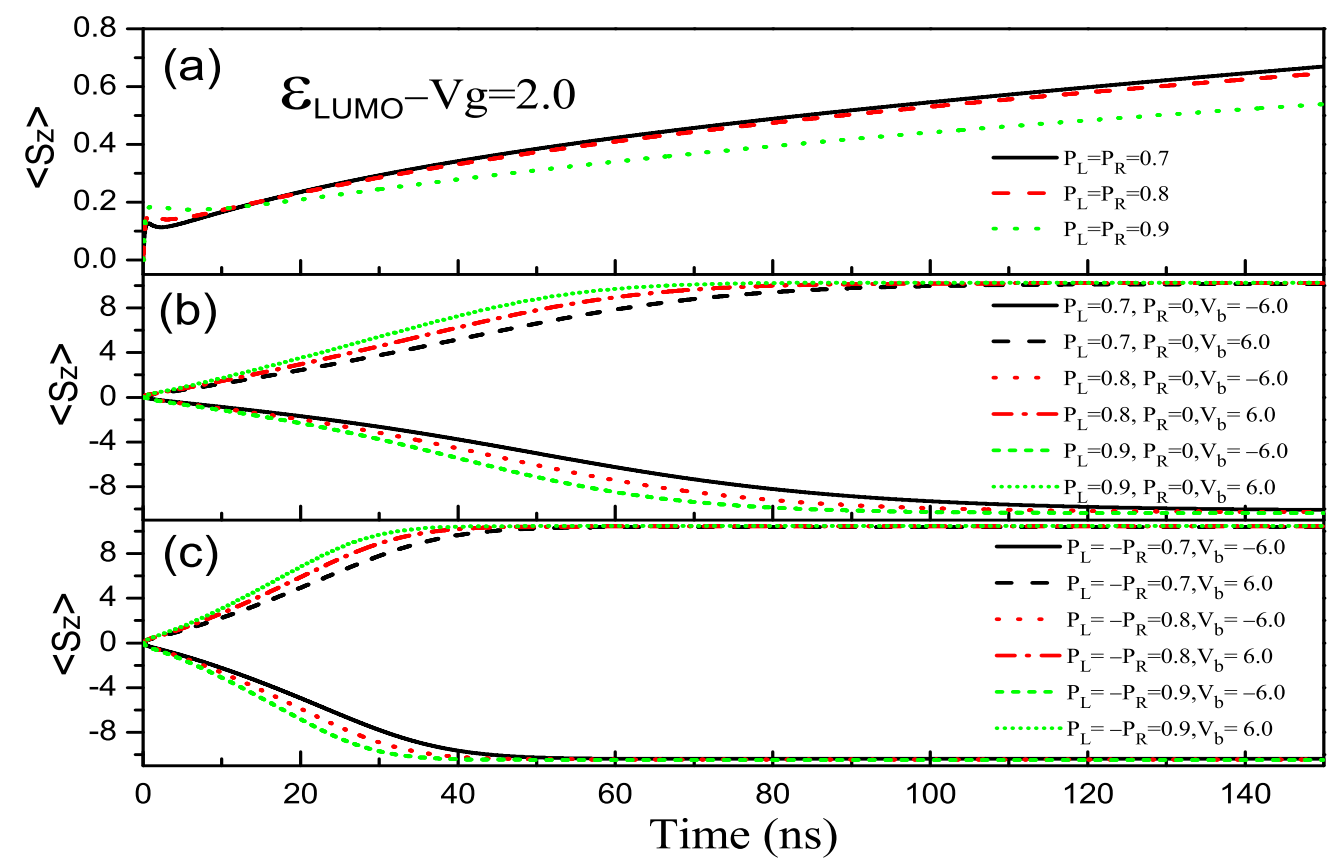

FIG. 2: (Color online) The average value of the $\left\langle S_{z}\right\rangle$ as a function of time for different values of $P_{L}$ and $P_{R}$ with $\varepsilon_{d}-e V_{g}=2.0$ and $V_{b}= \pm 6.0$. Here, we consider the three different configurations (a), $P_{L}=P_{R}=p$, (b), $P_{L}=p$ and $P_{R}=0$ and (c), $P_{L}=-P_{R}=p$. The SMM's parameters: $S=10, U=25, J=0.2, K_{1}=0.06, \Gamma_{L}=\Gamma_{R}=0.002$ and $k_{B} T=0.4$, where meV has been chosen as the unit of energy. 


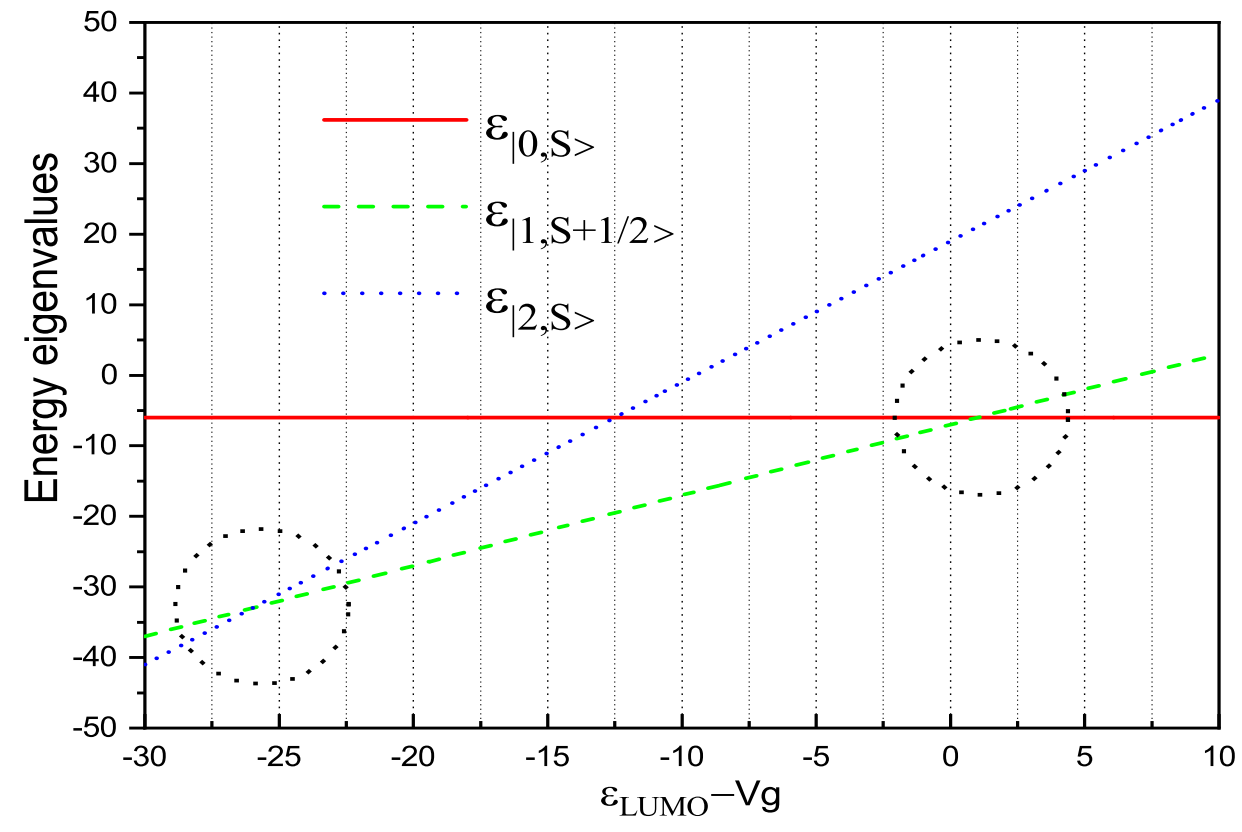

FIG. 3: (Color online) The minimum energy eigenvalues of the unoccupied, singly-occupied and doubly-occupied eigenstates as a function of $\varepsilon_{\mathrm{LUMO}}-e V_{g}$. The SMM's other parameters are the same as in Fig. 2. 

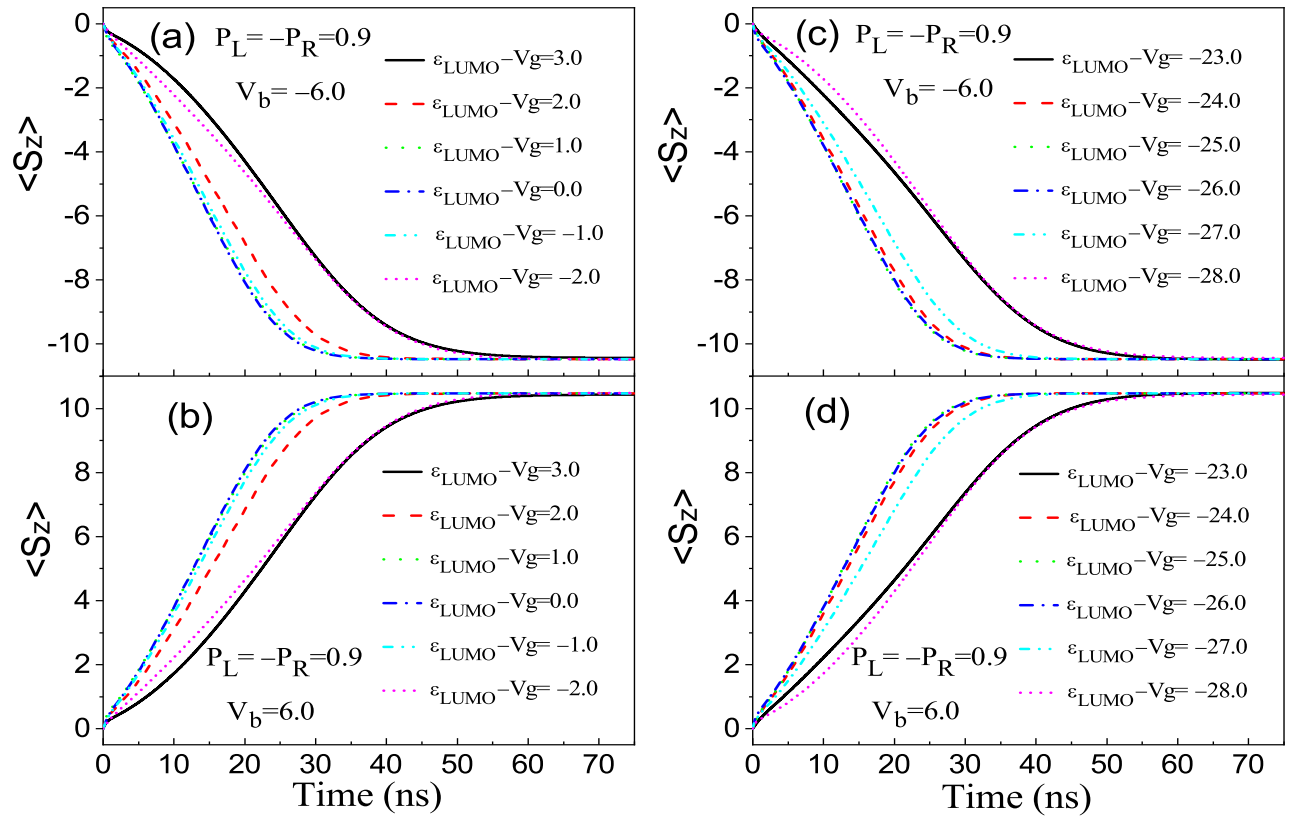

FIG. 4: (Color online) The average value of the $\left\langle S_{z}\right\rangle$ as a function of time for different values of $\varepsilon_{\mathrm{LUMO}}-e V_{g}$ with $P_{L}=-P_{R}=0.9$ and $\Gamma_{L}=\Gamma_{R}=0.002$. (a) and (c), $V_{b}=-6.0,(\mathrm{~b})$ and (d), $V_{b}=6.0$. The SMM's other parameters are the same as in Fig. 2. 

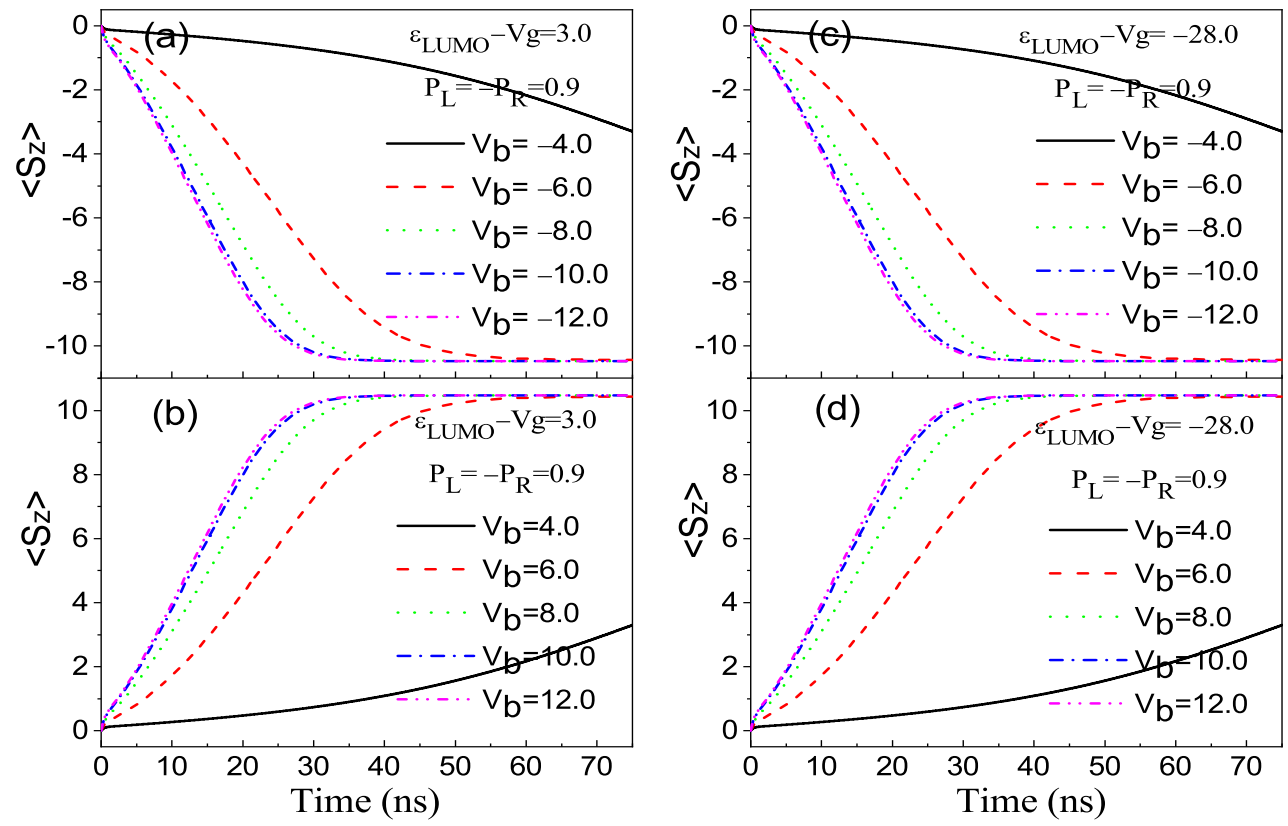

FIG. 5: (Color online) The average value of $\left\langle S_{z}\right\rangle$ as a function of time for different values of $V_{b}$ with $P_{L}=-P_{R}=0.9$ and $\Gamma_{L}=\Gamma_{R}=0.002$. (a) and (b), $\varepsilon_{\text {LUMO }}-e V_{g}=3.0$, (c) and (d), $\varepsilon_{\text {LUMO }}-e V_{g}=-28.0$. The SMM's other parameters are the same as in Fig. 2. 

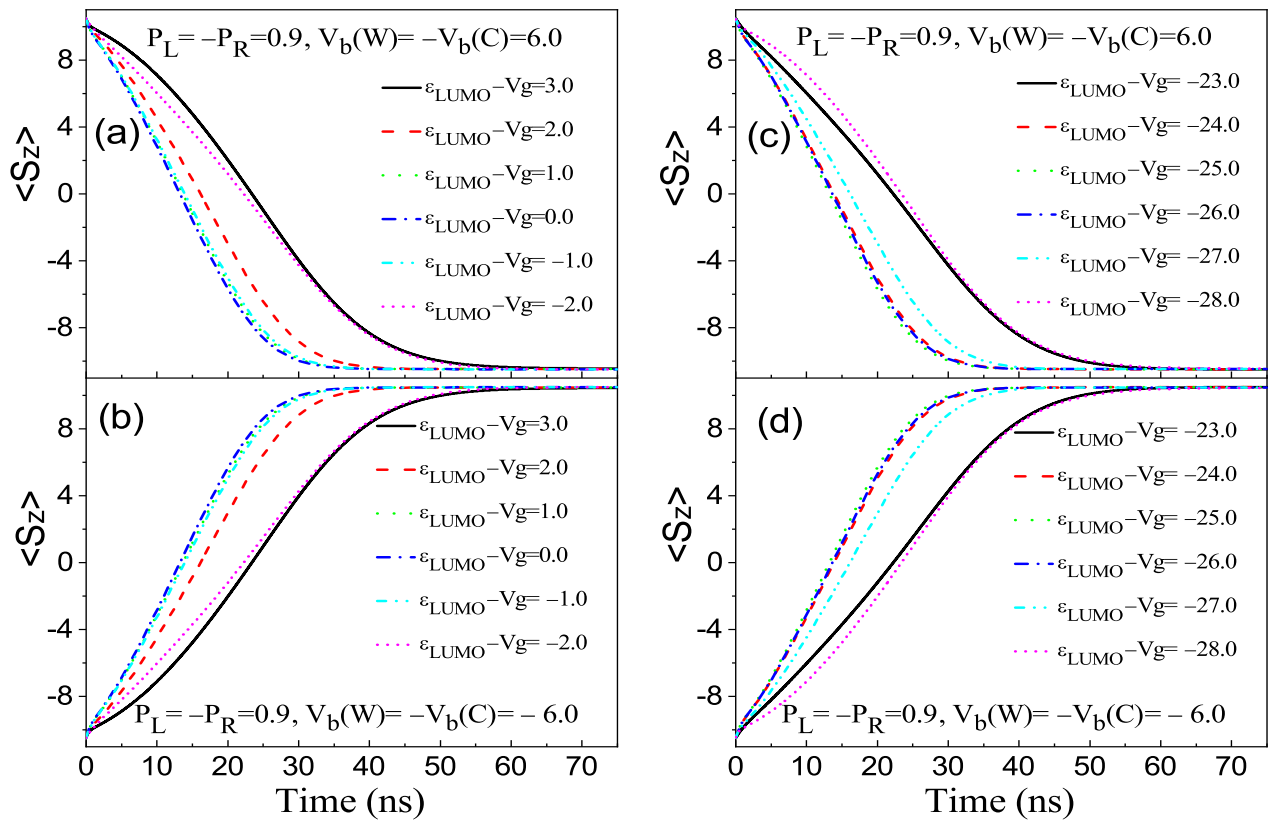

FIG. 6: (Color online) The average value of the $\left\langle S_{z}\right\rangle$ as a function of time for different values of $\varepsilon_{\text {LUMO }}-e V_{g}$ with $P_{L}=-P_{R}=0.9$ and $\Gamma_{L}=\Gamma_{R}=0.002$. Here, the initial spin states of the $\left\langle S_{z}\right\rangle_{\max }$ and the $\left\langle S_{z}\right\rangle_{\min }$ are chosen as the steady spin states in Fig. 4, and the magnitude of the controlling bias voltage $V_{b}(\mathrm{C})$ is chosen the same as that of the writing one $V_{b}(\mathrm{~W})$. (a) and (c), $V_{b}(\mathrm{~W})=-V_{b}(\mathrm{C})=6.0,(\mathrm{~b})$ and $(\mathrm{d}), V_{b}(\mathrm{~W})=-V_{b}(\mathrm{C})=-6.0$. The SMM's other parameters are the same as in Fig. 2. 

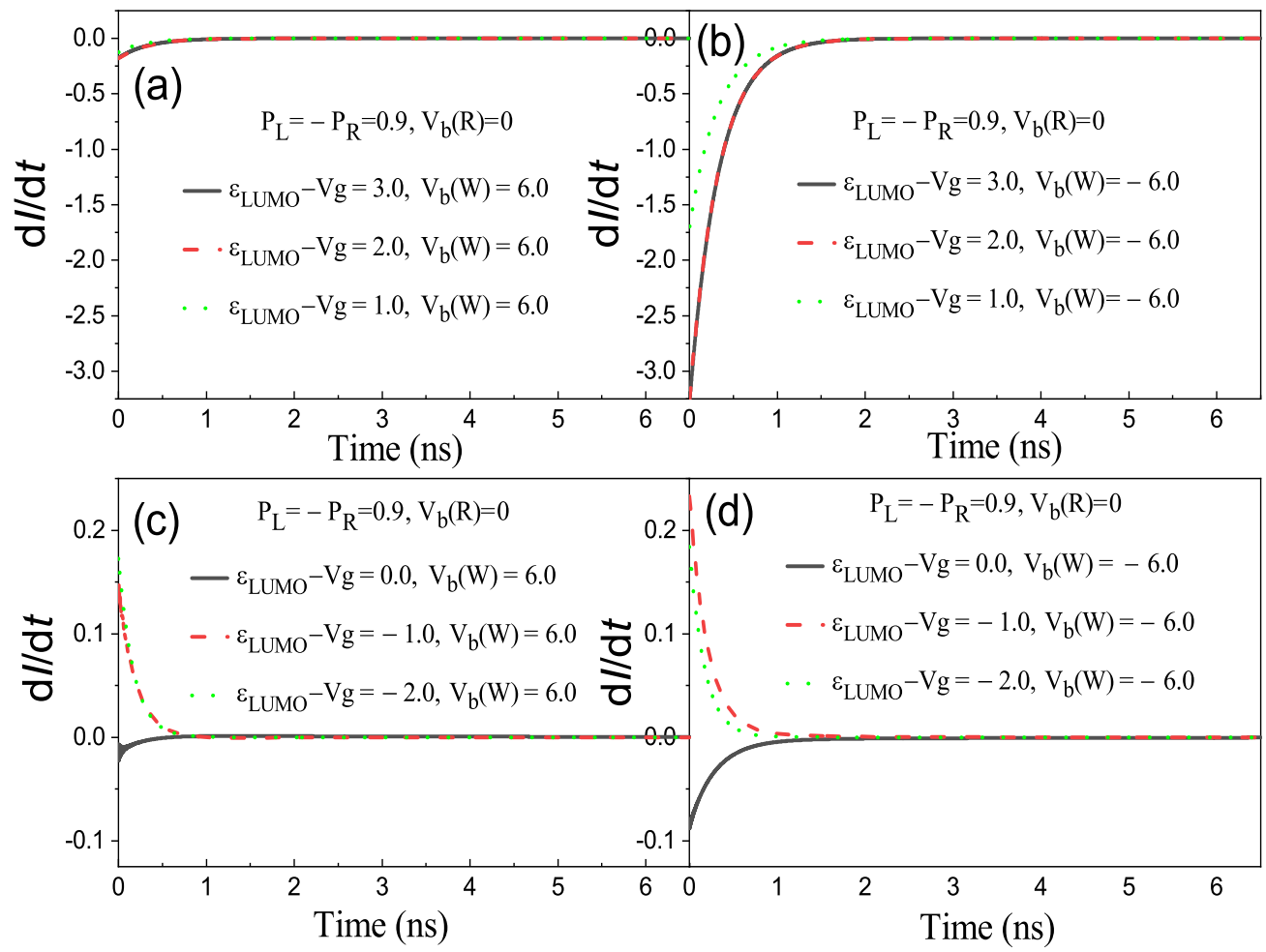

FIG. 7: (Color online) The time derivative of the transport current, namely, $d I / d t$, as a function of time for different spin states of stored information with $P_{L}=-P_{R}=0.9$ and $\Gamma_{L}=\Gamma_{R}=0.002$. (a) and (c), the spin-state of the $\left\langle S_{z}\right\rangle_{\max }$; (b) and (d), the spin-state of the $\left\langle S_{z}\right\rangle_{\min }$. The SMM's other parameters are the same as in Fig. 2. 

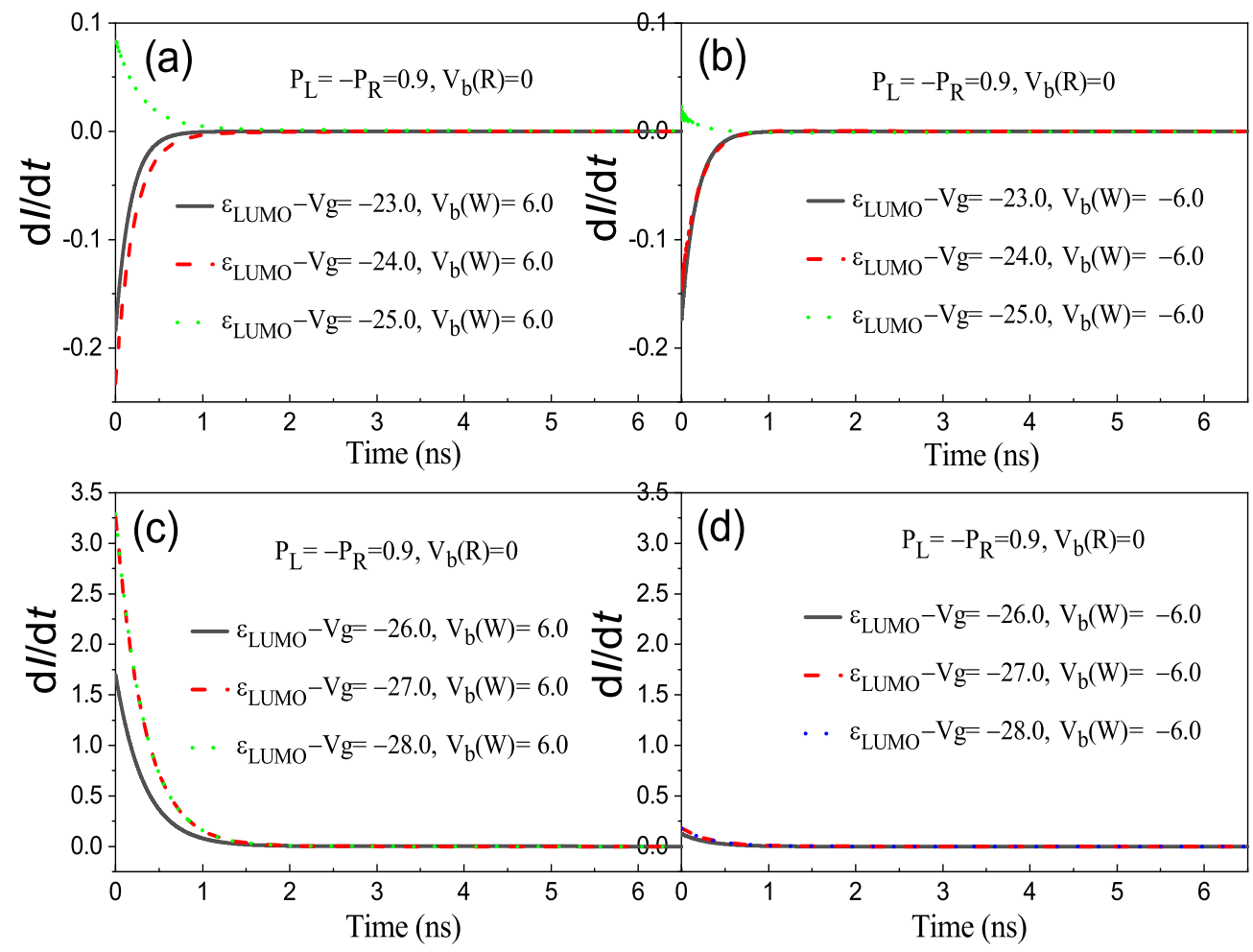

FIG. 8: (Color online) The time derivative of the transport current, namely, $d I / d t$, as a function of time for different spin states of stored information with $P_{L}=-P_{R}=0.9$ and $\Gamma_{L}=\Gamma_{R}=0.002$. (a) and (c), the spin-state of the $\left\langle S_{z}\right\rangle_{\max }$; (b) and (d), the spin-state of the $\left\langle S_{z}\right\rangle_{\min }$. The SMM's other parameters are the same as in Fig. 2. 

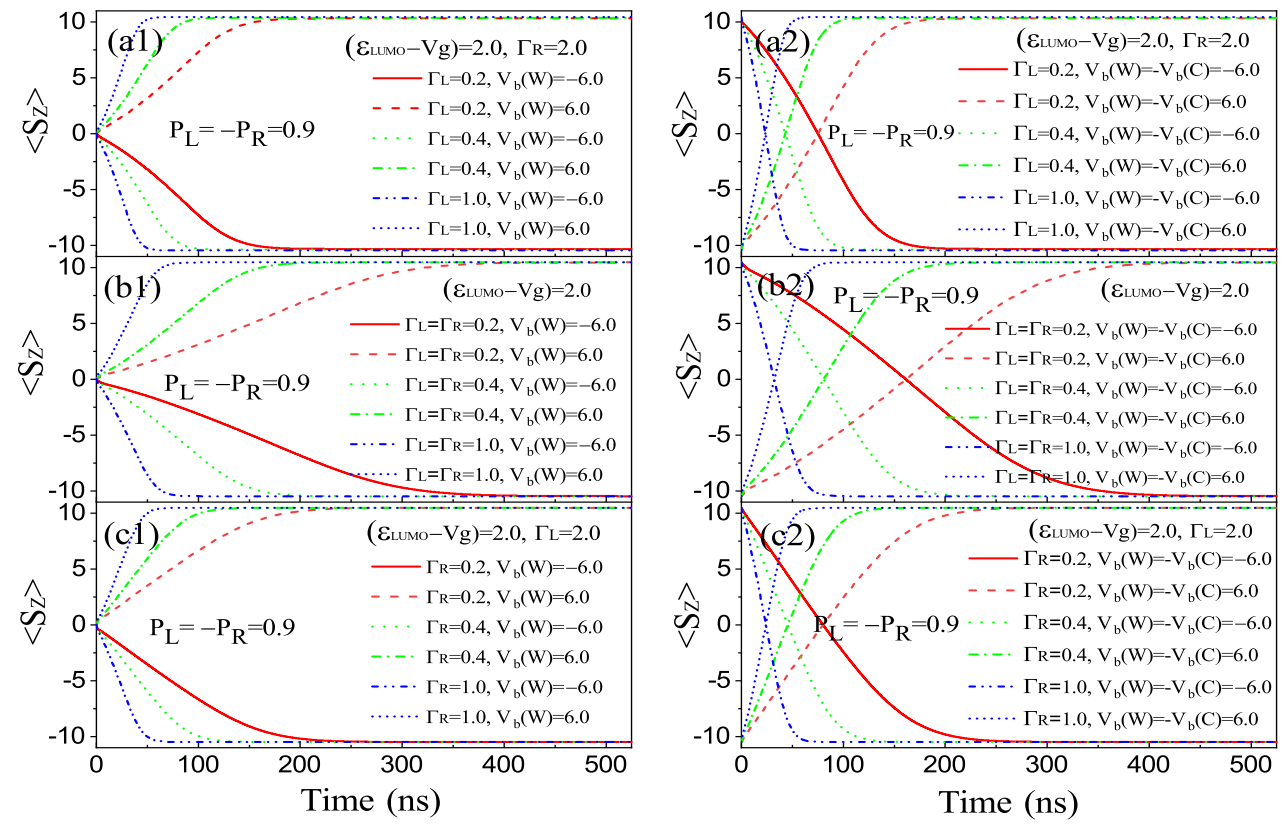

FIG. 9: (Color online) The average value of the $\left\langle S_{z}\right\rangle$ as a function of time for different values of the tunneling strength of and the asymmetry of SMM-electrode coupling. (a1), (b1) and (c1), $P_{L}=-P_{R}=0.9, \varepsilon_{\mathrm{LUMO}}-e V_{g}=2.0$ and $V_{b}(\mathrm{~W})= \pm 6.0 ;(\mathrm{a} 2),(\mathrm{b} 2)$ and $(\mathrm{c} 2), P_{L}=-P_{R}=0.9$, $\varepsilon_{\mathrm{LUMO}}-e V_{g}=2.0$ and $V_{b}(\mathrm{~W})=-V_{b}(\mathrm{C})=6.0$. (a1) and (a2), $\Gamma_{L}<\Gamma_{R},(\mathrm{~b} 1)$ and $(\mathrm{b} 2), \Gamma_{L}=\Gamma_{R}$, (c1) and (c2), $\Gamma_{L}>\Gamma_{R}$. The SMM's other parameters are the same as in Fig. 2. 

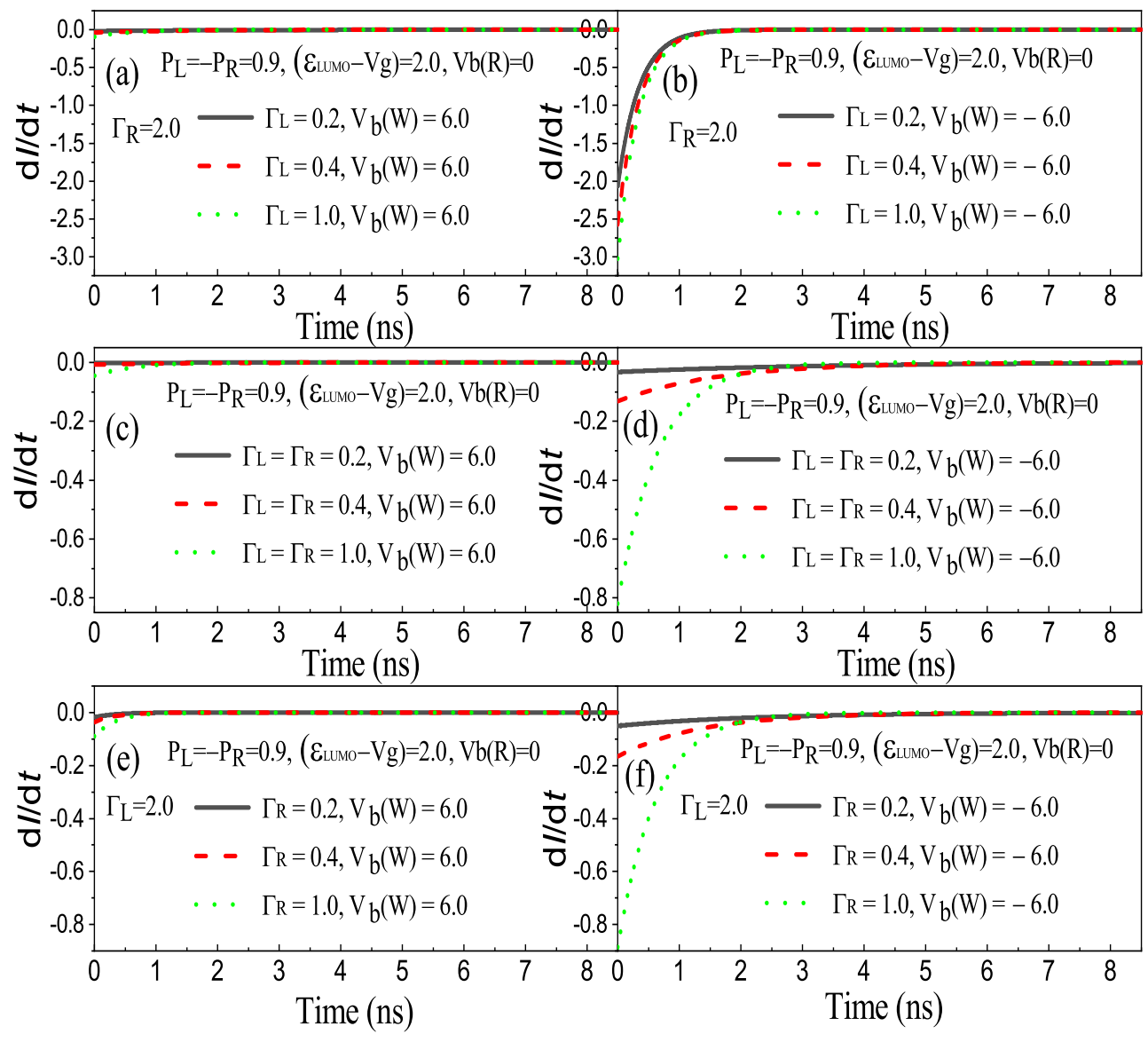

FIG. 10: (Color online) The time derivative of the transport current, namely, $d I / d t$, as a function of time for different spin states of stored information with $P_{L}=-P_{R}=0.9, \varepsilon_{\mathrm{LUMO}}-e V_{g}=2.0$ and $V_{b}(\mathrm{~W})= \pm 6.0$. (a1), (b1) and (c1) the spin-state of the $\left\langle S_{z}\right\rangle_{\max } ;(\mathrm{a} 2),(\mathrm{b} 2)$ and (c2) the spin-state of the $\left\langle S_{z}\right\rangle_{\max }$. (a1) and (a2), $\Gamma_{L}<\Gamma_{R}$, (b1) and (b2), $\Gamma_{L}=\Gamma_{R},(\mathrm{c} 1)$ and (c2), $\Gamma_{L}>\Gamma_{R}$. The SMM's other parameters are the same as in Fig. 2. 